\title{
Robust Pulmonary Nodule Segmentation in CT: Improving Performance for Juxtapleural Cases
}

\author{
K. Okada ${ }^{1}$, V. Ramesh ${ }^{1}$, A. Krishnan ${ }^{2}$, M. Singh ${ }^{1}$, and U. Akdemir ${ }^{1}$ \\ 1 Real-Time Vision \& Modeling Dept., Siemens Corporate Research, Princeton, USA \\ ${ }^{2}$ CAD Program, Siemens Medical Solutions, Malvern, USA
}

\begin{abstract}
Two novel methods are proposed for robust segmentation of pulmonary nodules in CT images. The proposed solutions locate and segment a nodule in a semi-automatic fashion with a marker indicating the target. The solutions are motivated for handling the difficulty to segment juxtapleural, or wall-attached, nodules by using only local information without a global lung segmentation. They are realized as extensions of the recently proposed robust Gaussian fitting approach. Algorithms based on i) $3 \mathrm{D}$ morphological opening with anisotropic structuring element and ii) extended mean shift with a Gaussian repelling prior are presented. They are empirically compared against the robust Gaussian fitting solution by using a large clinical high-resolution CT dataset. The results show $8 \%$ increase, resulting in $95 \%$ correct segmentation rate for the dataset.
\end{abstract}

\section{Introduction}

Pulmonary nodule segmentation is one of the major goals of the computer-aided diagnosis with the chest CT data (chest CAD 1,2,3]). A semi-automatic robust segmentation solution is required for realizing reliable volumetric measurement of nodules $[1,5]$, as an integral part of lung cancer screening and management.

Intensity-based segmentation solutions, such as local density maximum algorithm [6], have been successfully applied to the nodule segmentation problem. Although such solutions can be effective for solitary nodules, they cannot separate noduels from juxtaposed surrounding structures, such as walls and vessels, due to their similar intensity. Recently, to address this issue, more sophisticated approaches have been proposed to incorporate nodule-specific geometrical constraints 7,8. However, juxtapleural, or wall-attached, nodules still remain as a challenge because they can grossly violate such geometrical assumption and also appear frequently in practice. Another source of problem is rib bones which appear with high intensity values in CT. Such high-intensity regions near a given marker can bias the semi-automatic nodule center estimator. Robust segmentation of the juxtapleural cases can be addressed in two approaches: a) global lung or rib segmentation $[6,0,10,11$ and b) local non-target removal or avoidance 4. The former can be effective but also computationally complex and dependent on the accuracy of the whole-lung segmentation. The latter is more efficient than the former but more difficult to achieve high performance due to the limited amount of information available for the non-target structures. 
Addressing the above issue of the juxtapleural cases, this article proposes two novel 3D nodule segmentation solutions based on the local non-target removal and avoidance approach. The local analysis-based approach is preferred to the global one in the semi-automatic CAD context due to its efficiency. The first solution detects and removes the lung wall region within an input sub-volume by using 3D binary morphological opening operation. Similar approaches have been proposed 94, however our solution employs data-driven ellipsoidal 3D structuring element unlike others. The second solution is based on an extended mean shift framework incorporating a repeller (negative) prior which pushes the convergence away from a specific data point. This prior-constrained mean shift is used for correctly detecting the nodule center despite the presence of rib bones, thereby improving the segmentation accuracy without an explicit removal of the walls and ribs. Both proposed solutions are realized as extensions of the robust anisotropic Gaussian fitting solution [8], which is employed for deriving the ellipsoidal structuring element and the repeller prior.

This article is organized as follows. Section 2 summarizes the robust Gaussian fitting solution. Section 3 introduces the proposed solutions for handling the juxtapleural nodules. Section 4 presents the results of our performance validation with a large clinical CT dataset. Section 5 presents our conclusive remarks.

\section{Robust Anisotropic Gaussian Fitting}

This section summarizes the robust anisotropic Gaussian fitting algorithms proposed previously as a solution to the semi-automatic (one-click) 3D nodule segmentation problem 812. The one-click segmentation assumes that we are given a marker $\mathbf{x}_{p}$ indicating a rough location of the target nodule. Such marker can be provided from the radiologist's readings by eye-appraisal or the outcome of automatic nodule detection system [1314. For computational efficiency, the algorithm is usually applied to a sub-volume $V(\mathbf{x})$ centered at the marker and extracted from the 12-bit CT volume data $I(\mathbf{x}): \mathcal{R}_{+}^{3} \rightarrow \mathcal{R}_{+}$.

The algorithm results in a Gaussian function which fits the local intensity distribution of the target nodule best: $I(\mathbf{x}) \simeq \alpha \times\left.\Phi(\mathbf{x} ; \mathbf{u}, \boldsymbol{\Sigma})\right|_{\mathbf{x} \in \mathcal{S}}$ where $\Phi(\mathbf{x} ; \mathbf{u}, \boldsymbol{\Sigma})=|2 \pi \boldsymbol{\Sigma}|^{-1 / 2} \exp \left(-1 / 2(\mathbf{x}-\mathbf{u})^{t} \boldsymbol{\Sigma}^{-1}(\mathbf{x}-\mathbf{u})\right)$ is the anisotropic 3D Gaussian function. $\alpha$ is a positive magnitude factor. $\mathcal{S}$ is a local neighborhood forming a basin of attraction of the target. $\mathbf{u}$ is the fitted Gaussian mean indicating the estimated nodule center. $\boldsymbol{\Sigma}$ is the fitted Gaussian covariance matrix indicating the nodule's anisotropic spread. The nodule's 3D boundary is approximated by the $35 \%$ confidence ellipsoid of the fitted Gaussian, determined empirically.

The algorithm performs a multiscale analysis by considering a Gaussian scalespace of the input sub-volume. The Gaussian scale-space $L(\mathbf{x} ; h)$ is a solution of the diffusion equation $\partial_{h} L=\frac{1}{2} \nabla^{2} L$ with an initialization $L(\mathbf{x} ; 0)=I(\mathbf{x})$. Such a scale-space is defined by a convolution of $I(\mathbf{x})$ with a Gaussian kernel $K_{H}(\mathbf{x})$ with a bandwidth matrix $\mathbf{H}: L(\mathbf{x} ; h)=I(\mathbf{x}) * K_{H}(\mathbf{x} ; \mathbf{H}=h \mathbf{I})$. The algorithm considers a Gaussian scale-space constructed over a set of densely sampled discrete analysis scales $\left\{h_{k} \mid k=1, . ., K\right\}$. At each analysis scale, a fixed- 
scale robust analysis is performed for fitting an anisotropic Gaussian function in each scale space image. Given a set of estimated Gaussians, the most stable estimate across the scales determines the final outcome.

The fixed-scale analysis performs a robust Gaussian fitting with scale-space mean shift, a convergent weighted mean shift defined in the Gaussian scale-space,

$$
\mathbf{m}\left(\mathbf{x} ; \mathbf{H}_{k}\right)=\frac{\int \mathbf{x}^{\prime} K_{H}\left(\mathbf{x}-\mathbf{x}^{\prime} ; \mathbf{H}_{k}\right) I\left(\mathbf{x}^{\prime}\right) d \mathbf{x}^{\prime}}{\int K_{H}\left(\mathbf{x}-\mathbf{x}^{\prime} ; \mathbf{H}_{k}\right) I\left(\mathbf{x}^{\prime}\right) d \mathbf{x}^{\prime}}-\mathbf{x}=h_{k} \frac{\nabla L\left(\mathbf{x} ; h_{k}\right)}{L\left(\mathbf{x} ; h_{k}\right)}
$$

Gaussian mean $\mathbf{u}$ as the nodule center is estimated by the convergence of the majority of initial seeds sampled around $\mathbf{x}_{p}$. A set of new seeds are sampled around the estimated mean $\mathbf{u}$. The mean shift procedures are then performed from each seed. Gaussian covariance is estimated by a constrained least-squares solution of a linear system with unknown $\boldsymbol{\Sigma}$, constructed with mean shift vectors only along the convergent trajectories. The linear system can also be constructed with the response-normalized scale-space Hessian [12].

The multiscale analysis, given a set of Gaussians estimated at the analysis scales $\left\{\left(\mathbf{u}_{k}, \boldsymbol{\Sigma}_{k}\right)\right\}$, is realized by finding the most stable estimate among others using a divergence-based stability test. For this purpose, the algorithm employs the Jensen Shannon divergence (JSD) of three neighboring Gaussians computed at each analysis scale. Assuming the normal form of distributions, JSD can be expressed in the following simple form [15],

$$
J S D(k)=\frac{1}{2} \log \frac{\left|\frac{1}{3} \sum_{i=k-1}^{k+1} \boldsymbol{\Sigma}_{i}\right|}{\sqrt[3]{\prod_{i=k-1}^{k+1}\left|\boldsymbol{\Sigma}_{i}\right|}}+\frac{1}{2} \sum_{i=k-1}^{k+1}\left(\mathbf{u}_{i}-\mathbf{u}\right)^{t}\left(\sum_{i=k-1}^{k+1} \boldsymbol{\Sigma}_{i}\right)^{-1}\left(\mathbf{u}_{i}-\mathbf{u}\right)
$$

where $\mathbf{u}=\frac{1}{2} \sum_{k-1}^{k+1} \mathbf{u}_{i}$. The minimization of a JSD profile across the scales $h_{k}$ results in the most-stable-over-scales estimate $\left(\mathbf{u}^{*}, \boldsymbol{\Sigma}^{*}\right)[12$.

The robustness is due to two aspects of this algorithm. First, the fixed-scale Gaussian fitting solution performs robust model fitting with the outlier removal using the scale-space mean shift convergence analysis. This helps to mitigate the problem of juxtaposed neighboring structures. Second, the usage of the stabilitybased scale selection robustifies the fitting process even for intensity distributions that do not follow the Gaussian assumption well. This facilitates the effectiveness of the solution for segmenting clinically significant but technically challenging ground-glass nodules [8]16].

\section{Segmentation for Juxtapleural Cases}

Two proposed solutions described below extends the above robust Gaussian fitting solution for handling not only the solitary but also the juxtapleural cases. Both solutions first execute the robust Gaussian fitting. The resulting fitted Gaussian undergoes a goodness-of-fit test, analyzing chi-square errors between the data and fitted model, as well as a linear DC bias 8 . Only when the initial fitting results fail to pass the test, either of the following two solutions is invoked. 
Our pilot study resulted in following two empirical observations. First, the most of gross segmentation failures which can be detected by the goodness-of-fit test are due to the juxtapleural cases. Second, the initial fitted Gaussians for such failures tend to approximate the wall and rib structures. Exploiting these observations, we develop the segmentation solutions which employ the initial fitted Gaussian as an input to their process.

\subsection{Wall Removal by 3D Morphological Opening}

The input sub-volumes of the juxtapleural failure cases contain lung wall regions. Such wall regions appear typically as a large connected region with CT values higher than surrounding pulmonary parenchyma. The juxtapleural nodule will appear as a nodular structure partially embedded into the wall. The first solution explicitly removes the wall regions from the sub-volume using the morphological operation. Then the robust Gaussian fit is performed again on the wall-removed data, resulting in an improved segmentation of the target nodule. The algorithm consists of the following steps.

Wall Removal: Given the input $\left(V(\mathbf{x}), \mathbf{x}_{p}\right)$ and a fitted Gaussian $\left(\mathbf{u}^{*}, \mathbf{\Sigma}^{*}\right)$ failing the goodness-of-fit test, remove wall regions in $V(\mathbf{x})$, resulting in $V_{r}(\mathbf{x})$.

1. Binarize the input sub-volume $V(\mathbf{x})$ with an intensity threshold $t h_{1}$, resulting in a binarized sub-volume $B_{o}(\mathbf{x})$.

2. Compute the average diameter $d_{a v e}$ of the ellipsoid defined by $\boldsymbol{\Sigma}^{*}$.

3. Initialize a 3D structuring element: $\mathbf{E}=\boldsymbol{\Sigma}^{*}$ if $d_{\text {ave }}>t h_{2}$, otherwise $\mathbf{E}$ is set to a $3 \mathrm{D}$ ball with a fixed radius $r_{b}$.

4. Perform $3 \mathrm{D}$ binary morphological opening, resulting in smoothed volume $B_{s}(\mathbf{x})$ retaining only the large wall region: $B_{s}(\mathbf{x})=\left[B_{o}(\mathbf{x}) \ominus \mathbf{E}\right] \oplus \mathbf{E}$.

5. Perform a wall removal by masking $V(\mathbf{x})$ with the negative of $B_{s}(\mathbf{x})$ : $V_{r}(\mathbf{x})=V(\mathbf{x}) \times \operatorname{NOT}\left[B_{s}(\mathbf{x})\right]$

Nodule Segmentation: Perform the robust Gaussian fitting algorithm on $V_{r}(\mathbf{x})$ with $\mathbf{x}_{p}$, providing an improved nodule segmentation $\left(\mathbf{u}_{w r}, \boldsymbol{\Sigma}_{w r}\right)$.

(a)

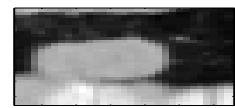

(b)

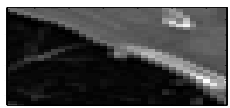

(c)

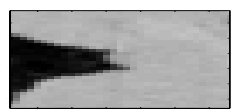

(d)

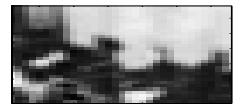

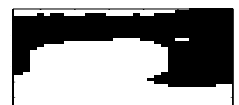
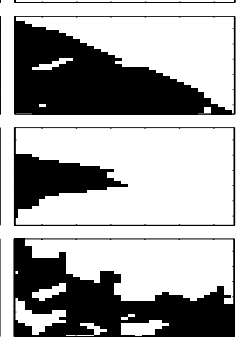
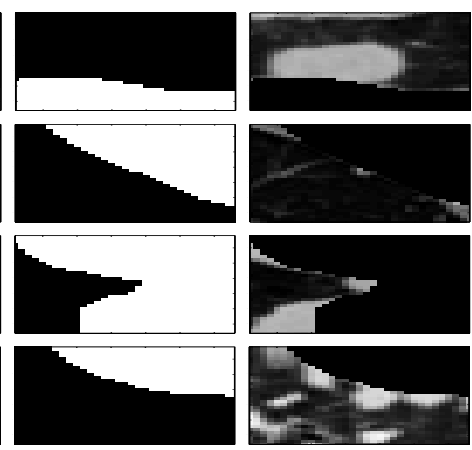

Fig. 1. Examples of the 3D morphological opening results shown in 2D cross-section images. From left to right column: input sub-volume $V(\mathbf{x})$, binarized sub-volume $B_{o}(\mathbf{x})$, smoothed sub-volume $B_{s}(\mathbf{x})$, wall-removed sub-volume $V_{r}(\mathbf{x})$. 
This algorithm utilizes a data-dependent ellipsoidal structuring element estimated for each data unlike the similar approach with a disc-like element [7. Our experimental results later show that this algorithm effectively reduces the segmentation failures due to the juxtapleural cases. Figure 1illustrates some examples of the morphological opening applied to the real CT data. It shows that the operation effectively removes the walls with large (1a), small (1b), heavilyembedded (1c), and irregular (1d) nodules. Note that this was achieved without using a set of structuring elements of different sizes.

\subsection{Mean Shift Constrained by Gaussian Repelling Prior}

The second solution is proposed for detecting the nodule center correctly without an explicit removal of the walls and ribs despite the presence of rib bones.

The prior-constrained mean shift incorporates a spatial prior information to the data-driven mean shift analysis. Suppose that the robust Gaussian fitting is performed on the sub-volume $V(\mathbf{x})$, resulting in the nodule center and spread estimate $\left(\mathbf{u}^{*}, \boldsymbol{\Sigma}^{*}\right)$. This fitted Gaussian can be interpreted as the normal probability distribution $Q(\mathbf{x})$ indicating a likelihood of $\mathbf{x}$ being the estimated center,

$$
Q(\mathbf{x})=\mathcal{N}\left(\mathbf{x} ; \mathbf{u}^{*}, \mathbf{\Sigma}^{*}\right)=\left|2 \pi \mathbf{\Sigma}^{*}\right|^{-1 / 2} \exp \left(-\frac{1}{2}\left(\mathbf{x}-\mathbf{u}^{*}\right)^{t} \mathbf{\Sigma}^{*-1}\left(\mathbf{x}-\mathbf{u}^{*}\right)\right)
$$

Suppose next that this estimate failed the goodness-of-fit test. This indicates that the estimated location $\mathbf{u}^{*}$ is not at the center of the target nodule and that the estimated spread $\Sigma^{*}$ roughly expresses the extent of the (rib/wall) structure which falsely attracted the mean shift convergence away from the true nodule center. Our main idea here is to re-estimate the nodule center with the constrained mean shift whose convergence is biased by the knowledge of $Q(\mathbf{x})$ so as to pushes the convergence away from the failed estimate $\mathbf{u}^{*}$.

To incorporate such a repelling (negative) prior, we consider resampling, or associating weights to, available data $I(\mathbf{x})$ to denote the notion that some data points are more likely to occur than others. We define such prior-induced positive weights by a negative of $\mathrm{Q}(\mathrm{x})$,

$$
w_{Q}(\mathbf{x})=1-\left|2 \pi \mathbf{\Sigma}^{*}\right|^{1 / 2} Q(\mathbf{x})
$$

Incorporating the negative prior leads to the following resampled scale-space $\tilde{L}(\mathbf{x} ; h)$ expressed in the discretized data space,

$$
\tilde{L}(\mathbf{x} ; h)=\sum_{i=1}^{N} w_{Q}\left(\mathbf{x}_{i}\right) I\left(\mathbf{x}_{i}\right) K_{h}\left(\mathbf{x}-\mathbf{x}_{i}\right)
$$

We call the mean shift $\mathbf{m}_{r}(\mathbf{x} ; h, Q)$ that is convergent to a mode in $\tilde{L}(\mathbf{x} ; h)$ negative prior-constrained scale-space mean shift. It is defined by,

$$
\mathbf{m}_{r}(\mathbf{x} ; h, Q)=\frac{\sum_{i} \mathbf{x}_{i} K_{h}\left(\mathbf{x}-\mathbf{x}_{i}\right) I\left(\mathbf{x}_{i}\right) w_{Q}\left(\mathbf{x}_{i}\right)}{\sum_{i} K_{h}\left(\mathbf{x}-\mathbf{x}_{i}\right) I\left(\mathbf{x}_{i}\right) w_{Q}\left(\mathbf{x}_{i}\right)}-\mathbf{x}
$$


Its convergence property is maintained because $w_{Q}\left(\mathbf{x}_{i}\right) \geq 0 \forall \mathbf{x}_{i}$.

A new Gaussian fitting solution is constructed by replacing the original scalespace mean shift (1) by this prior-constrained mean shift (6) in the robust fitting algorithm described in Section 2, Given an initial Gaussian $\left(\mathbf{u}^{*}, \boldsymbol{\Sigma}^{*}\right)$ failing the goodness-of-fit test, this new solution with $\mathbf{m}_{r}(\mathbf{x} ; h, Q)$ is executed on the original data $V(\mathbf{x})$, resulting in an improved segmentation with $\left(\mathbf{u}_{m s}, \boldsymbol{\Sigma}_{m s}\right)$.

\section{Experimental Results}

We compare the segmentation results of the two proposed methods against the baseline robust Gaussian fitting solution. We use a clinical HRCT dataset, consisting of 39 patients with 1312 nodules whose size ranges from $1 \mathrm{~mm}$ to $30 \mathrm{~mm}$ in diameter. The markers are provided by certified radiologists' eye-appraisal.

Table 1. Segmentation performance of the robust Gaussian fitting solution. GOF: the goodness-of-fit test. TP: accepted correct estimates. FN: rejected correct estimates. TN: rejected false estimates. FP: accepted false estimates.

\begin{tabular}{l|r|r|r}
\hline Classif. & \# Cases (\%) & GOF & \# Cases (\%) \\
\hline \hline Correct & $1156(88.1)$ & TP & $1095(83.5)$ \\
& & FN & $61(4.6)$ \\
\hline Failure & $156(11.9)$ & TN & $123(9.4)$ \\
& & FP & $33(2.5)$ \\
\hline
\end{tabular}

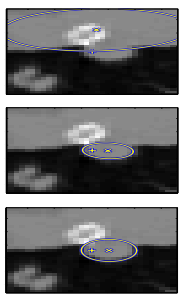

(a)

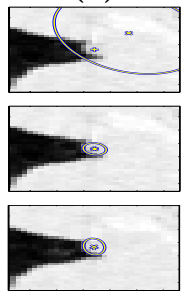

(g)

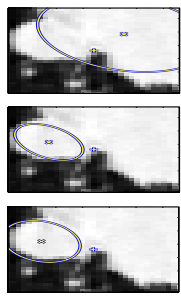

(b)

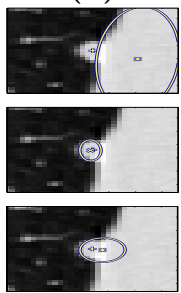

(h)
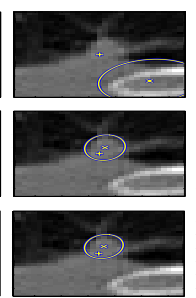

(c)

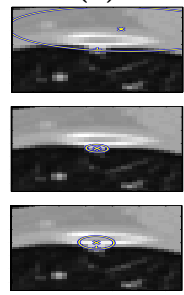

(i)

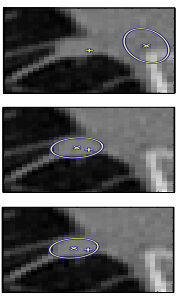

(d)

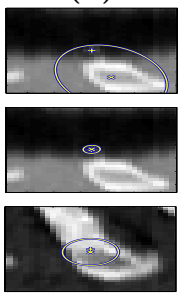

(j)

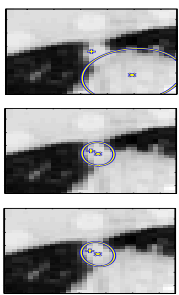

(e)

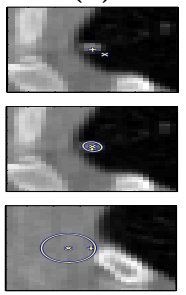

(k)

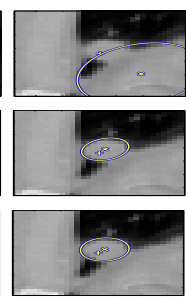

(f)

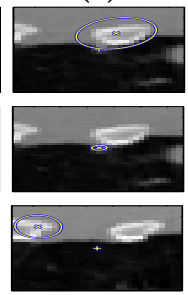

(1)

Fig. 2. Twelve examples of the 3D nodule segmentation results. 1st and 4 th rows: $\left(\mathbf{u}^{*}, \boldsymbol{\Sigma}^{*}\right)$ by the robust Gaussian fitting of Section 2. 2nd and 5th rows: $\left(\mathbf{u}_{w r}, \boldsymbol{\Sigma}_{w r}\right)$ by the morphological opening of Section 3.1 3rd and 6th rows: $\left(\mathbf{u}_{m s}, \boldsymbol{\Sigma}_{m s}\right)$ by the prior-constrained mean shift of Section 3.2 . 
$V(\mathbf{x})$ is set to $33 \times 33 \times 33$ in voxels centered at the markers. The correct/failure classification of the segmentation results are given by the agreement of experts by eye-appraisal. The implementation of the systems follows the parameter settings in 8] with an additional 2-layer Gaussian pyramid for handling larger nodules. For the morphological opening, we set $t h_{1}=500$ for the normalized intensity $I(\mathbf{x}) \in[0,4095], r_{b}=14$, and $t h_{2}=16.6$ in voxels, determined empirically. The prior-constrained mean shift does not have a free parameters to be tuned.

Table 1 summarizes the quantitative performance of the segmentation by the baseline robust Gaussian fitting solution. Together with the goodness-of-fit test, $88.1 \%$ and $9.4 \%$ resulted as true positives (TP) and negatives (TN). Among the 123 true negative cases, 108 were visually confirmed to be juxtapleural cases. The proposed nodule segmentation systems are tested with the $123 \mathrm{TN}$ cases.

Figure 2 shows some illustrative examples. Both methods successfully segmented the difficult juxtapleural cases that failed initially: large (2a-b), irregular (2c-d), and heavily-embedded (2e-h). The morphological opening-based solution (WallRemove: WR) segmented the small nodules better than the negative priorconstrained mean shift solution (MeanShift: MS) as shown in (2i-l). Incorporating more negative priors in MS by iterating the whole procedure can improve some failure cases that are surrounded by multiple distractors (2l).

The quantitative performance comparison is summarized in table 2. The results indicate that WR $(71.5 \%)$ performs better than MS (34.1\%), especially for the small juxtapleural cases. WR thus improves the overall segmentation performance from $88.1 \%$ to $94.8 \%$. Although MS's improvement was much lower than that of WR, there are some cases in which MS performs better than WR, as shown in Figure 3. When the nodules are attached to, or influenced by, non-

Table 2. Quantitative comparison of the two proposed segmentation solutions

\begin{tabular}{c|r|r}
\hline Classif. & MeanShift & WallRemove \\
\hline \hline Correct & $42(34.1)$ & $88(71.5)$ \\
\hline Failure & $81(65.9)$ & $35(28.5)$ \\
\hline Tot. Corr. & $1198(91.3)$ & $1239(94.8)$ \\
\hline
\end{tabular}
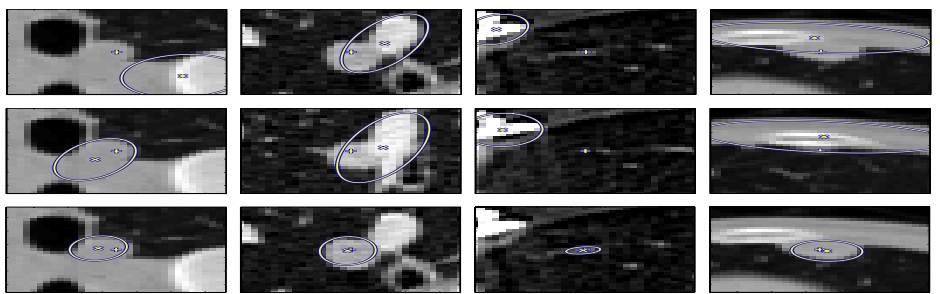

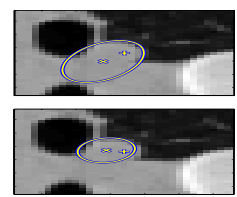

(a)

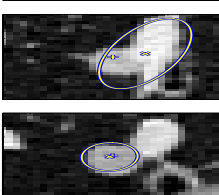

(b)

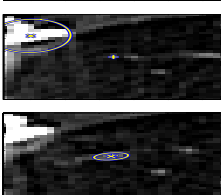

(c)

(d)

Fig. 3. Four example cases where the prior-constrained mean shift-based solution works better than the solution with wall removal using the morphological opening. From top to bottom row: initial fitted Gaussian $\left(\mathbf{u}^{*}, \boldsymbol{\Sigma}^{*}\right)$, after morphological opening $\left(\mathbf{u}_{w r}, \boldsymbol{\Sigma}_{w r}\right)$, with prior-constrained mean shift $\left(\mathbf{u}_{m s}, \boldsymbol{\Sigma}_{m s}\right)$. 
wall structures (3a-c), the morphological opening cannot be effective thus MS performs better. There are also several cases where a very large nodule was attached to a thin part of lung wall (3d). Such a case will result in over-estimation of the structuring element thus a failure of the wall removal.

\section{Conclusions}

We proposed two novel 3D nodule segmentation solutions which improve performance for the difficult juxtapleural cases. The morphological opening-based (WR) and prior-constrained mean shift-based (MS) solutions, extended from the robust Gaussian fitting approach, are evaluated with a large clinical CT dataset. The validation results show that i) they can effectively segment the juxtapleural cases, ii) WR performs better than MS for the small juxtapleural cases, and iii) MS performs better than WR for the cases attached to non-wall structures. Toward our goal of the volumetric measurement of nodules, the accuracy by our methods is limited due to the ellipsoidal boundary approximation. However, further improvement of segmentation quality is possible by incorporating a nonparametric segmentation with a Gaussian prior derived by using the proposed methods [17. Developing such an accurate non-parametric system together with the proposed solutions remains our future work.

\section{References}

1. Reeves, A.P., Kostis, W.J.: Computer-aided diagnosis of small pulmonary nodules. Seminars in Ultrasound, CT, and MRI 21 (2000) 116-128

2. van Ginneken, B., ter Harr Romeny, B.M., Viergever, M.A.: Computer-aided diagnosis in chest radiography: A survey. IEEE Trans. Med. Imag. 20 (2001) 1228-1241

3. Ko, J.P., Naidich, D.P.: Computer-aided diagnosis and the evaluation of lung disease. J. Thorac Imag. 19 (2004) 136-155

4. Ko, J.P., et al.: Small pulmonary nodules: Volume measurement at chest CT phantom study. Radiology 228 (2003) 864-870

5. Wormanns, D., et al.: Volumetric measurements of pulmonary nodules at multi-row detector CT : in vivo reproducibility. Eur. Radiol. 14 (2004) 86-92

6. Zhao, B., et al.: Two-dimensional multi-criterion segmentation of pulmonary nodules on helical CT images. IEEE Trans. Med. Imag. 22 (2003) 1259-1274

7. Kostis, W.J., Reeves, A.P., Yankelevitz, D.F., Henschke, C.I.: Three-dimensional segmentation and growth-rate estimation of small pulmonary nodules in helical CT images. IEEE Trans. Med. Imag. 22 (2003) 1259-1274

8. Okada, K., Comaniciu, D., Krishnan, A.: Robust anisotropic Gaussian fitting for volumetric characterization of pulmonary nodules in multislice CT. IEEE Trans. Med. Imag. 24 (2005) 409-423

9. Armato, S., et al.: Computerized detection of pulmonary nodules on CT scans. RadioGraphics 19 (1999) 1303-1311

10. Wang, P., O'Dell, W.: Automated detection of juxta-pleural lung nodules in chest CT using lung contour corrected by anatomic landmarks. In: AAPM. (2004)

11. Shen, H., Liang, L., Shao, M., Qing, S.: Tracing based segmentation for the labeling of individual rib structures in chest CT volume data. In: MICCAI. (2004) 
12. Okada, K., Comaniciu, D., Krishnan, A.: Scale selection for anisotropic scale-space: Application to volumetric tumor characterization. In: IEEE Conf. Comput. Vision and Pat. Recog. (2004)

13. Lee, Y., Hara, T., Fujita, H., Itoh, S., Ishigaki, T.: Automated detection of pulmonary nodules in helical CT images based on an improved template-matching technique. IEEE Trans. Med. Imag. 20 (2001) 595-604

14. Novak, C., Shen, H., Odry, B., Ko, J., Naidich, D.: System for automatic detection of lung nodules exhibiting growth. In: SPIE Med. Imag. (2004)

15. Comaniciu, D.: An algorithm for data-driven bandwidth selection. IEEE Trans. Pat. Anal. Mach. Intell. 25 (2003) 281-288

16. Henschke, C.I., et al.: CT screening for lung cancer: frequency and significance of part-solid and non-solid nodules. AJR Am. J. Roentgenol. 178 (2002) 1053-1057

17. Okada, K., Comaniciu, D., Krishnan, A.: Robust 3D segmentation of pulmonary nodules in multislice CT images. In: MICCAI, Saint Malo (2004) 